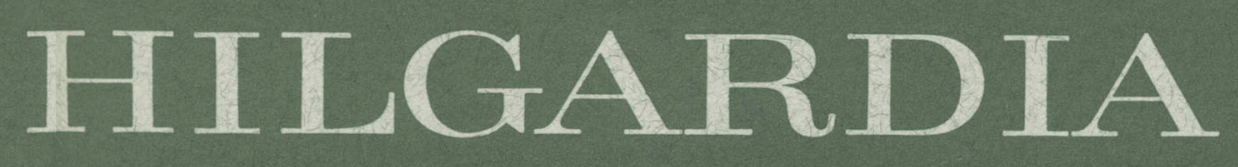

A JOURNAL OF AGRICULTURAL SCIENCE PUBLISHED BY THE CALIFORNIA AGRICULTURAL EXPERIMENT STATION

Volume 38, Number 4 March, 1967

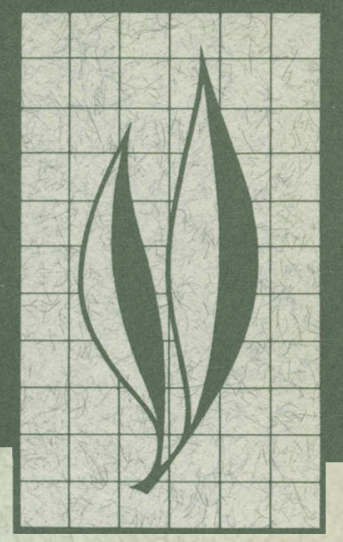

\title{
A Model for Simulating Photosynthesis in Plant Communities
}

W. G. Duncan, R. S. Loomis, W. A. Williams, and R. Hanau 


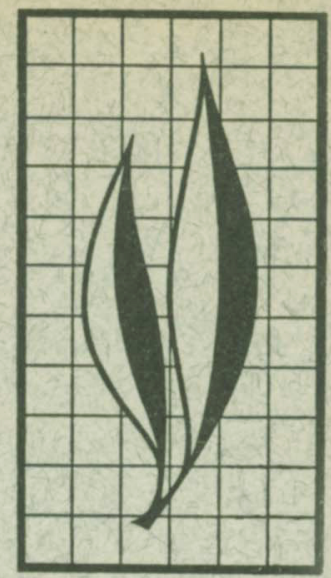

In this paper, the amount of photosynthesis accomplished by a canopy of leaves is predicted from the properties of individual leaves. Theories are developed for the penetration of direct and diffuse sunlight through a foliage composed of many layers of leaves, each having known orientation, area, reflection and transmission characteristics. From this, the illumination of each leaf at a particular time of day (sun elevation) and thus its photosynthesis, is determined. Hourly observations are summed for estimates of daily photosynthesis by plant communities.

An immense number of calculations are required in such a model, but an IBM 7044-type computer can complete them in about six seconds. Solutions to a number of real and hypothetical problems are presented. These provide new insights into the photosynthetic behavior of plant communities.

\section{THE AUTHORS:}

W. G. Duncan is Associate Professor of Agronomy, University of Kentucky.

R. S. Loomis is Associate Professor of Agronomy, Davis.

W. A. Williams is Professor of Agronomy, Davis.

R. Hanau is Professor of Physics, University of Kentucky. 


\section{A Model for Simulating Photosynthesis in Plant Communities ${ }^{1}$}

\section{INTRODUCTION}

THE PRIMARY synthesis of dry matter in all ecosystems is accomplished through the photosynthetic activities of green plants. While a great deal has been learned about the capabilities of individual leaves to assimilate carbon under controlled conditions, much less is known about the rate and efficiency of this process as it applies to plant communities.

Even in the carefully managed communities characteristic of intensive agriculture, the complexity of the interrelated variables-leaf and sun angles, spectral characteristics of direct and diffuse skylight, distribution of leaf area in space and time, photosynthetic functions of leaves, carbon dioxide gradients, and the like-has thwarted attempts at analysis. Some progress has been made in following the overall carbon dioxide exchange of sample communities in field chambers (Thomas and Hill, 1949; Moss, Musgrave and Lemon, $1961)^{2}$ and by aerodynamic analysis with transfer equations (Monteith, 1962; Lemon, 1960). Each of these approaches has its own limitations, however, and like the more classical technique of periodic sampling for dry matter production (Watson, 1958), fails to provide information on the contributions of individual leaves to performance of the whole community.

Experiments with whole communities which have utilized Watson's (1958) concept of leaf area index have underlined the importance of foliage configuration as a major determinant of net productivity (see the discussion by Loomis and Williams, 1963). For example, Watson and Witts (1959) compared the domesticated sugar beet with a wild type, the sea beet. The greater efficiency of the cultivated sugar beet in dense stands seemed to be associated with a more upright leaf habit, whereas extensive mutual shading seemed to be the key to the poor performance of the prostrate, rosette-leaved sea beet strains. Thus far, however, it has not been possible, despite some admirable attempts (Watson and Witts, 1959; Nichiporovich, 1961), to state confidently what morphological forms of plants and what architecture of the foliage canopy might be most efficient in intercepting and utilizing solar radiation.

Like Wit (1965) and Monteith (1965), we riewed the problem in two of its simpler aspects-one dealing with the geometry of leaf display and light re-

\footnotetext{
${ }^{1}$ Submitted February 7, 1966. This research was completed during the 1964-1965 academic year while the senior author was on leave from the University of Kentucky. It was supported in part by grants from the National Science Foundation to K. E. F. Watt, University of California, Davis (GE 8135) and to W. A. Williams and R. S. Loomis (GB 4192).

2 See "Literature Cited" for citations referred to in the text by author and date.
} 
ception, and the other with the physiological attributes of the leaves in different portions of the foliage. Wit and Monteith took empirical laws established from measurements made of individual plants and of natural plant communities and environments as their starting point, and generalized these into mathematical forms for a wide range of problems.

Our approach was to construct a model whose variables (in purely physical or mathematical terms) could be given any values. The model-thus quite flexible in its application-was designed for a computer of IBM class 7044 , or its equivalent, and can be solved readily and rapidly to yield information about almost any community and environment it simulates.

The principal physiological information needed for any approach is the characteristic photosynthetic light-response curve of leaves adapted to each layer of the foliage canopy. Light-response curves (photosynthetic rate of individual leaves as a function of light intensity) may be fitted rather closely by several functions, including that of a rectangular hyperbola:

$$
P=\frac{P_{\max } I}{I+k}-R
$$

where $P_{\max }$ is the asymptotic rate of photosynthesis; $I$ is the light intensity; $R$ is the dark respiration, and $k$ is a constant equal to $I$ at $\frac{P_{\max }}{2}$.

Wit (1965) assumed that $P_{\max }$ and $k$ decline linearly with depth in the canopy; but other authors have ignored the problem (Davidson and Philip, 1958), or have assumed that a single generalized curve will suffice to represent all leaves of a canopy at a given time. Work by Ludwig, Saeki and Evans (1965) has shown that the light response of lower, shaded leaves of cotton may be significantly different than that of the upper, well illuminated leaves. Such differences suggest the need for incorporating enough flexibility in any model to allow for variation in the light-response curve used from layer to layer in the foliage.

One then needs to know the amount of light received by each leaf in the canopy. Monsi and Saeki (1953) and Kasanga and Monsi (1954) were among the first to point out that sunlight is diminished as it proceeds into a dense foliage canopy. They showed that the attenuation is approximately exponential and thus analogous to BouguerLambert's law for light absorption in homogenous media. This same function is also obtained theoretically in Appendix B of this paper. Bouguer-Lambert's law was usefully $\epsilon$ mployed also by Monsi and Saeki (1953) and Davidson and Philip (1958) in their models of photosynthesis in plant communities, which predicted that for a given light condition, there is an optimum leaf area for community photosynthesis. Increasing leaf area up to the optimum leaf area index increases the rate of production of organic matter, but increasing leaf area beyond the optimum causes a decline, presumably the result of the mutual shading of leaves and the increased respiratory burden. Data from experiments with kale (Watson, 1959), subterranean clover (Davidson and Donald, 1958; Black, 1963), and rice (Takeda, 1961) have been corroborative. But experiments with mixed pasture (Brougham, 1956), sugar beet (Watson, 1958), and corn (Williams, Loomis and Lepley, 1965b) have not displayed an optimum leaf area index.

Monsi and Saeki (1953), Monsi (1960), and Verhagen, Wilson, and Britten (1963) have applied several other light extinction functions to characterize the light environment within various types of foliage. Verhagen $e t$ al. (1963) defined an "ideal" foliage canopy as one in which the extinction coefficient changes with depth in such a manner that average intensity of light 
is the same for every leaf, regardless of its depth in the canopy. This, of course, is not attained in nature, but their analysis does point to the possibility of manipulating the architecture of foliage to favor a more efficient light absorption pattern.

Monteith (1965) used a binomial expansion to express light attenuation with depth. For the $n$th leaf strata:

$$
\frac{I_{n}}{I_{o}}-\{s+(1-s) T\}_{n}
$$

Where $s$ is the fraction of total incident radiation which is not intercepted by leaf, and $T$ is the mean transmission coefficient of leaves.

With appropriate estimates of $s, T$, and for the constants of the lightresponse curves, he was able to calculate dry matter production-leaf area curves similar to the optimum or plateau-type curves obtained in field experiments.
Wit (1959) was the first to use a model in which the absorption of diffuse skylight was treated separately from direct radiation. In his model the foliage was treated as randomly oriented leaves in a single layer sufficiently thick to intercept all the light. Given the solar elevation, totals of direct and diffuse radiation, and a Blackman-style lightresponse curve approximating the behavior of many crop species, tables of potential productivity for various latitudes and times of the year were computed. A satisfactory fit to experimental data was obtained with this model by Stanhill (1962) with alfalfa and by Alberda and Sibma (1962) with pasture grass; while Williams, Loomis and Lepley (1965a) observed that the model substantially underestimated their experimental values for photosynthesis in corn under high intensity radiation (736 cal $\left./ \mathrm{cm}^{2} \mathrm{day}\right)$.

\section{DEVELOPMENT OF A MODEL FOR SIMULATING PHOTOSYNTHESIS IN PLANT COMMUNITIES}

Many compromises and modifications must be accepted in order to make a model simple enough to be solved with the available mathematical tools in a reasonable time-but it should not be so simple that biases or misleading relationships would occur. Our main objective was to develop a model to show qualitatively the effects of changes in any of the variables. Quantitative simulation of a real plant community was a desirable but secondary objective.

\section{Components of the plant community}

One part of the model consists of the plant community description. This may be visualized as many small, flat leaves dispersed in space and unsupported by stems. These leaves are divided by horizontal planes into layers, so that the vertical distance of any leaf above the surface of the soil can be described.
The following properties of plant leaves are used in the model as controlled variables:

\section{Leaf area}

2. Leaf angle

3. Vertical position

4. Light reflected from leaves

5. Light transmitted through leaves

6 . The physiological relationship between illumination and photosynthesis (light-response curve)

The physical properties of the leaves. The use of small flat leaves in the model is justified by the assumption that leaves of any shape or size can be approximated by dividing them into a large number of small flat segments. The size of each segment is not specified, except that they are small enough to be randomly distributed, regardless of how small the total leaf area might be within a layer. 
Leaf angles vary within layers of most plant communities. Where leaves are curved, the range might cover a full $90^{\circ}$ from vertical to horizontal in a single layer. However, since the number of layers that may be used is unlimited, a single layer in a real plant community may be approximated in the model by using several layers, each containing the area of all leaves within some range of angles. For example, the real leaves may be measured and divided into classes with the leaf areas within each $15^{\circ}$. angle class being grouped together. Each angle class then could be used to make up a sublayer of the model, so that six layers of the model could represent one layer of the real plant community. The order of the sublayers, whether the more nearly horizontal leaves were on the top or bottom sublayer would make some difference, but it would be small except, perhaps, with large areas of leaf per layer. Those situations could be evaluated empirically from hypothetical data with the simulation program to learn whether corrective measures should be introduced.

Leaves may be randomly arranged within layers of some species or with some cropping practices, but not in others. The model is simpler if randomness is assumed, although other assumptions might be quite possible if the type and degree of leaf organization to be simulated were known. The same is true of directional orientation. We have assumed that leaves grow equally in all directions around the individual stems, as might be the case with tobacco or with plants with $180^{\circ}$ phyllotaxis, such as corn, which usually have no favored directional orientation. The applicability of the model is limited to plant communities whose leaves have no tropic movements to significantly disturb random orientation, such as the sun-facing of many of the clovers.

The leaf area and position are described as leaf area index (LAI) within each layer. LAI is defined as the area of leaves (one surface) divided by the area of land over which the leaf areas are measured. However, some leaves are rugose or very rough. How should they be measured; how should other green and presumably photosynthetic surfaces, such as leaf sheaths and stems, be considered? What allowance can be made for the heads of small grains?

The small flat leaves of the model in this study can simulate any of these surfaces, if their areas and angles can be described. As arbitrary guidelines we suggest that reasonable allowance be made for marked rugosity or rippling, but not for ordinary surface roughness. For sheaths, stems, grain heads, and other structures that are green, the whole area exposed to light might be used, if it is between $60^{\circ}$ and $90^{\circ}$ (vertical); but only half of the LAI should be used if the area is between $60^{\circ}$ and the $0^{\circ}$ (horizontal). Fortunately, the area of green surfaces other than leaves is relatively small, in most cases, so that the error introduced by such approximations is likely to be insignificant.

Leaves are assumed to be vertically separated enough in relation to their width to permit uniform penetration of skylight. This, together with the definition of sunlight as coming from a point source, permits the actual height of the plant community to be disregarded. This will be discussed in more detail later. If the leaves of plants to be simulated are closely overlapping, so that some of their surfaces are permanently shaded from both sunlight and skylight, the measured leaf area should be appropriately reduced.

Leaves vary widely in their light-reflecting properties, but the actual light flux reflected is usually small in relation to the total incident light. Its significance in the photosynthesis of the plant community is that some light is lost back towards the sky in the crop albedo and that some is transferred by reflec- 
tion from leaves at the top, which may be near light saturation, to the lower, more shaded leaves, where it may be used more efficiently. In the model, as is approximately true for many real plants, leaves are assumed to act as lambertian surfaces, reflecting light nondirectionally. The model is less accurate for very glossy, smooth leaves which reflect light in a highly directional manner. Transmitted light is assumed to penetrate further into the community as completely diffuse light.

The light-response curve for single leaves. The effect of illumination in terms of photosynthesis depends on the light-response curve of single leaves. This may vary among leaves of the same plant with age or position in the canopy or with carbon dioxide or moisture status. It varies widely among species in maximum rates attainable. While many data are well fitted by rectangular hyperbolae, other curves may also be possible (Rabinowitch, 1951). Computation of the model requires that some light-response curve or curves be specified as well as the criteria for choosing among the curves for each layer.

An important aspect of the relationship between illumination and photosynthesis is the dark respiration. Unpublished work by the senior author supported by other evidence (Rabinowitch, 1945; and Ludwig et al., 1965) indicates that values for dark respiration found in the literature cannot be applied to real plant situations without some qualifications, since the rate of respiration in the dark may vary for the same leaf by a factor of two depending on its previous illumination history. Shaded leaves, and presumably the lower leaves in dense stands, have lower rates of respiration than recently illuminated leaves. This means that light curves may differ for upper and lower leaves.

In the present work, provision was made for two curves with the choice between them dependent on the level of diffuse light computed for the particular layer. At high levels of diffuse light, a curve obtained experimentally at 300 ppm carbon dioxide concentration with leaves adapted to high light intensities is used (Hesketh, 1963). At low levels, usually below 3 per cent of full sun, the same curve is used, but it is transposed vertically by a small amount to compensate for the low respiration rates and low light compensation values observed experimentally for shaded leaves. Ideally, light-response curves for such studies should be determined from several leaves, each adapted to the light intensity at which its rate is to be measured. However, such data are not available in the literature. In the program, a scparate light-response curve could be specified for each layer of leaves if desired.

Photosynthetic rates vary also in response to other environmental factors such as temperature, humidity and carbon dioxide concentration. These are not considered in the present model because not enough is known about how they vary within plant communities. However, in an ideal model, the concentration of carbon dioxide, for example, calculated in some manner for each layer, would be used as a basis for varying the light-response curve.

\section{Components of the plant environment}

Environment for the model consists of a point source of light simulating the sun, which may be at any elevation above the horizon, and a hemispherical sky. The variables of the environment are:

1. Elevation of the sun above the horizon

2. Solar intensity

3. Skylight brightness

The incident solar radiation. The azimuth angle of the sun is not necessary, since the leaves are randomly dispersed and are given uniform direc- 
tional orientation. While the description of the sun as a point source greatly simplifies the computations, it also limits the ecological situations which can be simulated. If the sun were a point source of light, all shadows would be sharp, and all leaf area would be either in full sunlight or illuminated only by skylight and by light reflected by or transmitted through leaves. This assumption avoids the need to consider size or shape of leaves or their vertical separation. This, in turn, permits the use of small flat leaves to simulate all the different shapes and sizes of leaves actually found.

These assumptions mean that the model cannot accurately describe tall plants with great separation between leaves, or pine trees, or plants such as asparagus with needle-like leaves, where shadows are not sharp. For most grasses, shrubs and agricultural crops, however, leaf separation in relation to leaf size is probably great enough to avoid serious errors. This is more likely to be true, since such errors tend to be self-compensating. Penumbral effects would increase the area of sunflecks, but at the same time, illumination of the lighted area would be decreased as relative to calculations based on a point source.

Skylight is assumed to come from a hemisphere of uniform brightness. Actually, the sky is more luminous nearer the sun than away from it. Also, the sky is often dotted with white clouds, each of which may act as a strong light source. The haze band close to the horizon and other irregularities also reduce uniformity of illumination. This does not introduce as much error as might be thought, however, because the model assumes uniform orientation and random distribution of leaves. In effect, this averages light from all compass directions, so the brighter southern sky in the Northern Hemisphere is averaged in with the northern sky. Brightness for different elevations above the horizon, however, is not averaged. The sky is assumed to be divided into zones of equal area by horizontal planes. Each zone is calculated independently, so that if information about sky brightness in any particular problem were available, the program could be modified by changing the number of zones and their relative brightness. For most cases, we would agree with Wit (1965) that, at present, the only reasonable assumption is that the sky is uniform in brightness.

Reflected and transmitted light. Plant leaves differ widely in the proportion of light which they reflect and transmit. Some plants have leaves that differ in both texture and color on the two sides. Some are completely opaque: others transmit a significant amount of light. Some leaves shine almost as if they were polished, while others look more like velvet. Many plants have leaves with their lower surfaces lighter in color or less reflective than the upper surfaces. These variations must have ecological significance which could be easily studied by this model with minor modification. These factors, according to Wit (1965), can be ignored, but we believe that the distinctions between reflected and transmitted light should be approximated as closely as possible.

In the model, all leaves are assumed to reflect light as a lambertian surface with light flux being proportional to the cosine of the angle of incidence. Similarly, transmitted light is assumed to be completely diffuse. With these assumptions, leaves radiate diffuse light in all directions, but not equally, since the greatest flux is normal to the leaves. Thus, the leaf angle would affect the flux and direction of both reflected and transmitted light.

For computation, the light reflected from leaves within any layer must be divided into three parts, the light reflected upward, the light reflected 
downward, and the light added to the effective illumination within the layer itself. Probably only a small bias is introduced if the reflected light is merely added to the skylight illumination within the layer-although light moving downward to darker layers is used more efficiently than light moving upward, which is absorbed by more highly illuminated leaves or escapes from the canopy entirely.

Reflected or transmitted light is considered as a hemisphere around each point on a leaf surface. The light transmitted upward or downward is that contained within a circular cone inside the hemisphere of some arbitrary halfangle, whose axis is vertical and passes through the point of the leaf surface. The most appropriate angle choice for the half-angle of the cone would be the one calculated for best agreement with the albedos of crop surfaces whose leaf areas, angles, and reflective properties were precisely known. In the almost total absence of such experimental data, it was found that a value of $35^{\circ}$ for the half-angle gave best agreement with accepted measurements of albedo and estimated leaf properties. This value has been used, therefore, in the absence of a better one.

The problem of calculating the part of the reflected or transmitted light to be found within such a cone is relatively simple for leaf angles of less than $55^{\circ}$ in the case of cones with $35^{\circ}$ half-angles. The mathematical equation developed by Hanau in Appendix B is:

$$
F=B \pi A \cos \alpha \sin ^{2} \beta
$$

where $B$ is the brightness of the surface; $A$, the area; $\alpha$, the leaf angle; $\beta$, the half-angle of the cone as measured between the axis and an element of the side; and $F$, the part of the light flux contained within the cone.

Once the flux of light moving up or down through the foliage has been estimated, the next step is to compute what part will pass through the layers above and below, and what part will be intercepted. This involved some simplifying assumptions that were justified by the comparatively minor importance of this light flux in relation to the whole. Light within such a $35^{\circ}$ half-angle cone, although actually moving at angles of up to $35^{\circ}$ from the vertical, is considered to be moving in a vertical direction, up or down. As such, the intercepting leaf area within each layer is found by multiplying the LAI by the cosine of the leaf angle. This area is taken as the part of the whole area that obstructs passage of the light. While this procedure overestimates the leaf area by ignoring self shading, it underestimates the length of the light path by assuming vertical movement.

Light moving down, from one layer to another, is reduced by the amount absorbed in passing through the next lower layer. This reduced light is added to the light originating in the lower layer from reflections and transmitted light, which move in the same direction. Light absorbed within each layer is added to the skylight that reaches the lower layer, plus its reflected light which is not included with the two $35^{\circ}$ cones. These computations result in increasing the diffuse illumination of leaves.

Computations of total illumination for each leaf layer. The leaves of the model are assumed to be illuminated by four distinct light sources: direct sunlight, skylight, reflection from other leaves, and transmission through them. Direct sunlight and skylight require two different mathematical treatments and theories. Reflected and transmitted light are treated as diffuse light, like skylight. Computation for these are described in the previous section. In the following discussion, the basis for treating direct and diffuse light will be followed by a description of how the methods are applied in actual computation of the model. 
1) Direct sunlight. Light from a point source at infinite distance is considered to be made up of parallel rays, and the proportion of such rays able to penetrate each foliage layer without being intercepted must be computed. The penetration of the light rays is a function of the area of leaves, their angle, and of the elevation of the sun above the horizon. The mathematical theory for the passage of inclined needles through foliage was developed by Reeve (1960), as the theory of inclined point quadrats. This work forms the basis for this part of the theory as outlined in Appendix A.

The $F^{\prime} / F$ ratio developed by Wilson (1960) and Reeve to express the probability of contact by an inclined needle is also the ratio between the actual area of a leaf and the shadow it would cast in the context of light rays. Clearly, the part of the light from a point source penetrating a layer of leaves would be a function of the area of shadow each leaf could cast.

The area of light passing through a layer of leaves cannot be reduced simply by the total shadow area of the leaves, because the shadows cast by some leaves will fall on other leaves within the same layer. More light will always pass through a layer than if the leaves were arranged in a mosaic. The assumption of random distribution of leaves within a layer permits use of the Poisson distribution equation to estimate the probability of penetration by rays of light. In this case, the mean probability of a rigid needle touching a single leaf is given by the following equation:

$$
P=\frac{a}{A} \frac{\left(F^{\prime} / F^{\prime}\right)_{j k}}{\sin k}
$$

or

$$
P=L A I \frac{\left(F^{\prime} / F\right)_{j k}}{\sin k}
$$

where $a$ is the area of the leaf; $A$, the area of land over which the leaf is measured; $\left(F^{\prime} / F\right)_{j k}$, the Wilson-Reeve $F^{\prime} / F$ ratio for leaf angle $j$ and sun angle $k$; and $\sin k$, the sine of the angle of elevation of the sun. Division by the sine of the solar elevation angle corrects for the length of the light path through the foliage layer. The ratio, $a / A$, is the $L A I$. The fraction of light passing through a foliage layer is:

$$
\begin{array}{r}
G=1-\left[\frac{P^{1} e^{-P}}{1 !}+\frac{P^{2} e^{-P}}{2 !}\right. \\
\left.+\frac{P^{3} e^{-P}}{3 !} \cdot \cdots\right]
\end{array}
$$

The first term in the parentheses gives the chance of a needle striking one leaf, the second of striking two leaves, and so on. We are interested in the chance of not striking any leaves, hence all other possibilities are subtracted from one. The algebraic sum of this series is:

$$
\begin{aligned}
G & =e^{-P} \\
\text { or } \quad G & \left.=e^{-\left[L A I\left(F^{\prime} / F\right)\right.}{ }_{j k} / \sin k\right]
\end{aligned}
$$

The area of light $I$ passing through such a foliage layer, where the area of light entering from above is $I_{o}$ is:

$$
I=I_{0} G
$$

or $\left.\quad I=I_{0} e^{-\left[L A I\left(F^{\prime} / F\right)\right.}{ }_{j k} / \sin k\right]$

This is the form of the equation for the Bouguer-Lambert's law, with constants expressed in terms of the variables of the problem. It is interesting that this simple derivation agrees with the experimental observations referred to in the literature review.

Note that when used for light from a point source, this equation gives the area of light penetrating each foliage layer. However, the effect is the same as if it computed flux penetrating, since the flux per unit area of light from a point source remains constant at all layers. The same equation is used to compute the penetration of diffuse skylight, but in that case, total flux rather than the area is computed. 
2) Skylight. The computation of light from skylight is based on Hanau's derivation (see Appendix B). With this method, the illumination of either or both surfaces of a leaf of any angle from any zone of the hemispherical sky can be calculated. The total skylight illumination of an unshaded leaf can then be expressed as the sum of the skylight from all sky zones.

All the light in a sky zone is assumed to come from its midpoint circle. Like the elevation of the sun, the relation of a zone's angle of elevation to the angle of leaves within a foliage layer is a basic factor in measuring the amount of light that penetrates the leaf layer. With some changes in meanings, the actual light flux penetrating from each zone is given by the same equation as for sunlight:

$$
\left.I=I_{0} e^{-\left[L A I\left(F^{\prime} / F\right)\right.}{ }_{j k} / \sin k\right]
$$

where $k$ is the elevation of any zone, and $I$ and $I_{0}$ is the light from that zone below and above the foliage layer.

This equation is solved for each zone, and the total value of skylight in the middle of each foliage layer is computed as the sum of the values of $I$ calculated for light from each sky zone.

The calculated illumination due to skylight is taken to be the sum of skylight on upper and lower leaf surfaces. Moss (1964) showed that this assumption would introduce little error in most cases. All leaves are assumed to be illuminated by skylight, and areas of leaves in direct sunlight are assumed to be illuminated by sunlight plus skylight.

\section{Computations for photosynthesis}

To actually compute photosynthesis, both the leaf area illuminated by sunlight plus diffuse light, and that area illuminated only by diffuse light within each layer, must be estimated. These factors are then given values in an equation expressing the relationship between illumination and photosynthesis.
To convert area of sunlight at a given brightness above and below a layer to estimated photosynthesis per unit of leaf area within the layer, it is necessary to consider the angular relationship between sun and leaf surface. This is done by successively rotating the leaf to six compass positions between $0^{\circ}$ and $180^{\circ}$ from the solar azimuth. At each position the illumination of the leaf surface is computed by multiplying the sun brightness, measured normally, by the cosine of the angle of incidence. The result is then added to the diffuse light illumination as computed for the midpoint of the layer. The resulting illumination, whether or not the front or back of the leaf is illuminated, is then computed in terms of milligrams of $\mathrm{CO}_{2}$ per square decimeter of leaf area per hour based on the light curve selected. The computations of the six positions are added and divided by six to give the average rate of photosynthesis for a unit of leaf area illuminated by sunlight plus diffuse light for the layer. Since symmetry is assumed, this average is the same as for the 12 positions from 0 to $360^{\circ}$.

To compute the area of sunlit leaves within each layer, the area of sunlight emerging from each layer is subtracted from the area entering. This must be corrected for the length of the light path to give the area in horizontal units, and then divided by the Wilson-Reeve $F^{\prime} / F$ factor. The equation for this is:

$$
A=A_{0}-A_{1}\left[\sin k /\left(F^{\prime} / F\right)_{j k}\right]
$$

where $A_{o}$ is the area of light at the top of the layer; $A_{1}$ is the area at the bottom of the layer; $k$ is the elevation of the sun; $j$ is the leaf angle; and $A$ is the area of leaves within a layer illuminated by the direct light of the sun. This value, multiplied by the average photosynthesis per unit area of sunlit leaves, gives the photosynthesis within the layer, of leaves in direct sunlight. 
The difference between the total LAI per layer and the area in direct sunlight, $A$, gives the area of leaves illuminated only by diffuse light. This area is then multiplied by the unit rate of photosynthesis computed for leaves in diffuse light in that layer to give the total photosynthesis for the leaves in the layer that are not in direct sunlight. The sum of photosynthesis computed for sunlit and shaded leaf area gives the rate of photosynthesis for the whole layer in units of $\mathrm{mg} \mathrm{CO} \mathrm{Cm}^{-2} \mathrm{hr}^{-1}$. This is converted to $\mathrm{gm} \mathrm{CO}_{2} \mathrm{~m}^{-2} \mathrm{hr}^{-1}$ by use of a suitable factor. The sum of such values for all layers gives the estimated photosynthesis for the community.

The particular program used is arranged to select the light-response curve for a particular layer of leaves on the basis of the foot-candles of diffuse light calculated for that layer. The computer uses one light-response curve above the transition light level, another below it. The transition level is given as part of the input data.

\section{CALCULATION PROCEDURES}

A typical problem for which the model may be used is the estimation of photosynthesis for each hour of the day with different values for one or more variables of the plant community or of the environment. For this, two sets of data cards are prepared, one set describing the environment and the other set describing the plant community. Typically, the environmental descriptions might consist of the sun elevations for each hour from dawn until solar noon for some latitude and date. Corresponding to each hour would be appropriate data for sunlight brightness as measured normally and of the illumination of a horizontal surface by skylight. In the absence of actual hourly observations, the afternoon might be assumed to be a replication of the period before solar noon; the results for the morning are simply multiplied by two.

The description of the plant community would consist of the number of layers chosen, the leaf angle and area within each layer, and information about characteristics of the leaves. In such a program, the computer would calculate the photosynthesis for each layer starting at the first hour of the morning. It would then find the total for all layers for that hour, add this to previous totals for an accumulative total, then proceed to repeat this for the next hour. This would be repeated until the computer received a signal in the data input that the last hour to be considered had been completed. At this point, the summaries would be printed along with information about the input data used, values assigned to variables and constants, and other information that identifies the problem for future reference.

The time required for computing the photosynthesis in a day, by hours, varies with the number of layers, the length of day, and other factors; but for typical problems, using the IBM 7044, about six seconds was used per calculated day. The program used for this study provided for printing out information about the illumination calculated for all layers, if called for in the output specifications. This was given in two forms which corresponded to two ways of measuring illumination within the plant community. One form corresponded to the reading that might be made by exposing a horizontal unshaded light meter, corrected to receive light from all directions, at a number of locations at the same level and averaging the results. The second form corresponded to the readings of a horizontal light meter shaded from direct sunlight. 


\section{EXAMPLES OF COMPUTER SIMULATIONS OF HYPOTHETICAL AND REAL PROBLEMS}

An accurate description of both plants and climate over some growing period is necessary in order to simulate a real plant community. With this information, the computer can calculate rates of photosynthesis for any time interval desired. However, to form an opinion about the accuracy of these results, one must have some actual measurements of growth rates in the plant community for the same intervals. There must also be some assurance that photosynthesis is not restricted in any way, and that all assumptions are valid. Even with extensive measurements and a great deal of care in selecting and laying out the experimental work, considerable uncertainty usually exists about respiration losses and other unknowns.

In contrast, it is easy to set up hypothetical problems with simple and clearly defined plant and climate de- scriptions. If the assumptions are simple enough, the relative accuracy of the computations may be judged as reliable. When the values of each of the variables are changed, the results should be in the expected direction and of the expected relative magnitude. Also, values for variables beyond anything attainable in nature should produce extreme results that are reasonable and consistent. If the model and computer program give reasonable answers under all these conditions, acceptable answers in more complex cases would seem probable.

In the problem sets that follow, the values or range of values of the nine variables are listed in tabular form with general comments about the procedure to be used. The first three hypothetical problems illustrate the versatility and the wide range of application of the model with nine plant and climate variables under control.

\section{Problem 1}

\section{Variables}

Plant:

1. Leaf area index for each layer

2. Leaf angle for each layer

3. Leaf reflectivity

4. Leaf transmissivity

5. Number of layers

6. Light curve for upper leaves

Climate:

1. Solar elevations

2. Solar intensity

\section{Skylight brightness}

\section{Values}

From 0.1 to 1.0

$0^{\circ}, 40^{\circ}$, and $80^{\circ}$

17 per cent of incident light

5 per cent of incident light

10

$P_{\max } K 120, P_{10,000} 60, R=2.0$

Appropriate for latitude $38^{\circ} \mathrm{N}$, July 1

Appropriate for clear day at above latitude, date and time

Appropriate for clear day at above latitude, date and time

For these and all problems that follow, 24-hour whole-plant respiration will be assumed to be 40 per cent of the total photosynthesis. All 10 layers are assumed to have the same LAI values and the same leaf angles, unless stated otherwise. 
Figure 1A shows the rates of photosynthesis per hour throughout a typical day for three different LAI values with all leaves at $0^{\circ}$ leaf angle (horizontal). Figure $1 \mathrm{~B}$ and $1 \mathrm{C}$ show the same LAI values with leaf angles of $40^{\circ}$ and $80^{\circ}$, respectively. Figure $1 \mathrm{C}$ is noteworthy because higher photosynthetic rates are shown to be associated with high leaf angles, especially during the midday period when the sun is high in the sky and when enough leaf area is available to intercept the light. Also interesting in figure $1 \mathrm{C}$ is the midday depression in photosynthetic rates with inadequate leaf area. Probably with high leaf angles and low LAI, the proportion of the total light intercepted at high sun elevations is low enough to more than compensate for the high efficiency per unit of leaf area. Where LAI is high, the combination of high total light interception and a high utilization efficiency gives high rates of photosynthesis per unit of land area. Monteith (1965) made a similar explanation; when his penetration factor " $s$ " is equated with leaf angle as used here, more horizontal leaves correspond to lower " $s$ " values. The values seem reasonably close to some given by Wit (1965).

It may be noted also that with the more horizontal leaves, photosynthetic rates per unit of land area actually decrease as LAI increases. Perhaps the respiration of lower leaves illuminated below their compensation points more than offset the slightly higher light interception associated with increased LAI. Or, as LAI increases, the canopy more nearly resembles a horizontal sheet, which would give less photosynthesis per unit of land area than an arrangement permitting more leaf area to be illuminated. This would be the case under some conditions, even if leaf respiration were zero.

The same factors are shown in a slightly different form in figure 2. It should be noted that at low LAI values, the more nearly horizontal leaves give the highest photosynthetic rates for the community. With LAI values above approximately 3.5, the higher leaf angles are more effective. This is similar to a figure by Monteith (1965) in which his term $s$ approximates the effect of varying leaf angles. In comparing his figures with figure 2 , there is some difference in the convexity of the curves, but this difference would be affected by the assumptions used for the illumination-photosynthesis curves of single leaves and for other factors.

\section{Variables}

Plant:

1. Leaf area index for each layer

2. Leaf area angle for each layer

Other variables and values same as in problem 1

Climate:

1. Solar elevations

\section{Solar intensity}

\section{Skylight brightness}

\section{Problem 2}

$$
\begin{aligned}
& 0.4 \\
& 40^{\circ}
\end{aligned}
$$

This problem illustrates the use of the model to estimate latitude and date effects on photosynthetic rates. In this particular problem the longer days of the more northern latitude are almost compensated for the higher sun angles and the

Appropriate for latitude $28^{\circ} \mathrm{N}$ and $48^{\circ}$ $\mathrm{N}$, July 1

Appropriate for clear days at the date and latitudes assumed

Same as above

\section{Values}




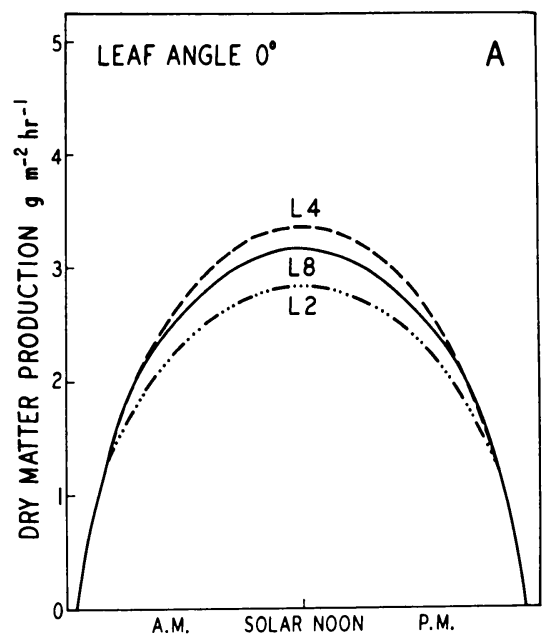

Fig. 1 (left, A, B, C). Computed hourly net photosynthesis in several plant communities with leaf area index (L) equal to 2,4 , or 8 and with leaf angle varying, (A) all leaves at $0^{\circ}$ (horizontal); (B) all leaves at $40^{\circ} ;$ (C) all leaves at $80^{\circ}$ (near vertical).

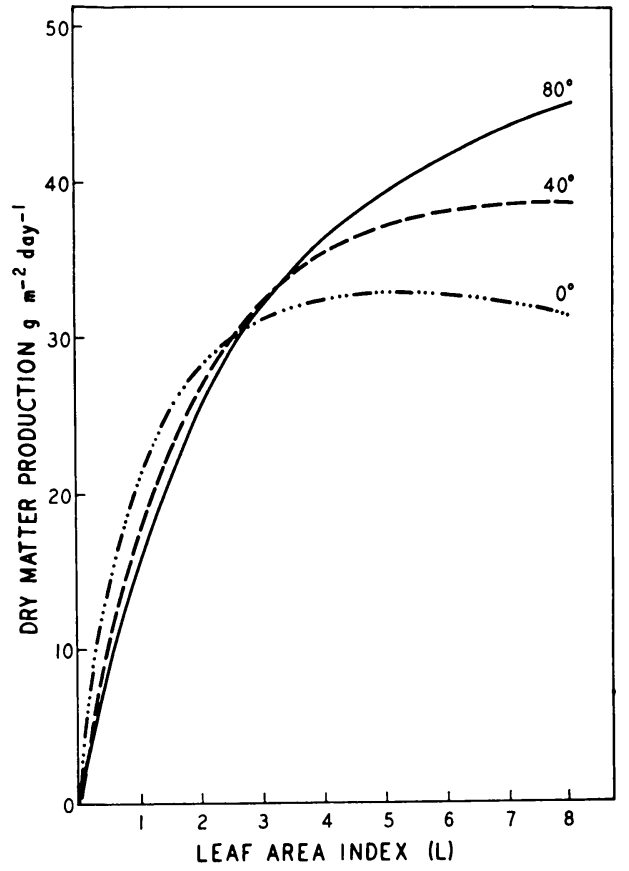

Fig. 2. Computed daily net photosynthesis in plant communities with uniform leaf angles of $0^{\circ}, 40^{\circ}$, and $80^{\circ}$, as a function of leaf area index.

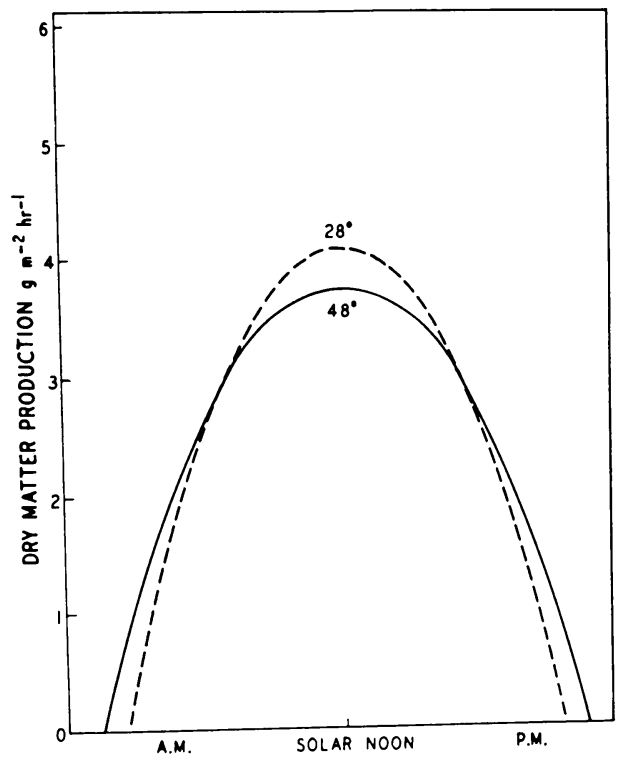

Fig. 3. Computed daily net photosynthesis in identical communities of maize at two latitudes. 
higher radiation intensity of southern latitudes (fig. 3). The computed totals were $35.4 \mathrm{gm}$ of dry matter per day for $48^{\circ}$ latitude, 35.2 for $28^{\circ}$. Different assumptions about dates or plant variables or cloudiness would produce quite different results.

\section{Variables}

Plant:

1. Leaf area index for each layer

2. Leaf angle for each layer

\section{Problem 3}

\section{Values}

Assume three leaf area distributions with LAI equal to 4.0: (1) each layer 0.4 ; (2) LAI per laver increasing stepwise from 0.2 for the top layer to 0.6 for the bottom layer, and (3) LAI per layer decreasing stepwise from 0.6 for the top layer to 0.2 for the bottom.

Assume three leaf angle distributions: (1) each layer at $45^{\circ}$; (2) decreasing by $10^{\circ}$ steps from $90^{\circ}$ for the top layer to $0^{\circ}$ for the bottom, and (3) increasing by $10^{\circ}$ steps from $0^{\circ}$ for the top layer to $90^{\circ}$ for the bottom.

All other plant and climate variables are the same as in problem 1.

The purpose of this problem is to illustrate the application of the model to the problem of studying the "leaf architecture" of plant communities. Different values for the other variables held constant in this problem would affect the relationships.

In problem 3 , it is interesting that the distribution of leaf area had no significance in total photosynthesis for the stand, as long as the leaf angles were the same at all layers (table 1). Results were significantly different, however, when the leaf angles were changed.
TABLE 1

SIMULATED PHOTOSYNTHESIS AS AFFECTED BY FOLIAGE ARCHITECTURE*

\begin{tabular}{|c|c|c|c|}
\hline \multirow[b]{2}{*}{$\begin{array}{l}\text { Leaf angle } \\
\text { distribution }\end{array}$} & \multicolumn{3}{|c|}{ Leaf area distribution } \\
\hline & $\begin{array}{c}\text { Each } \\
\text { layer } 0.4\end{array}$ & $\begin{array}{c}\text { Top } \\
\text { layer } 0.2 \\
\text { Lowest } \\
\text { layer } 0.6\end{array}$ & $\begin{array}{c}\text { Top } \\
\text { layer } 0.6 \\
\text { Lowest } \\
\text { layer } 0.2\end{array}$ \\
\hline & \multicolumn{3}{|c|}{ gm. of dry matter per sq. meter per day } \\
\hline Each layer $45^{\circ}$. & 34.0 & 33.8 & 34.2 \\
\hline $\left.\begin{array}{l}\text { Top layer } 90^{\circ} \ldots \\
\text { Lowest layer } 0^{\circ} \ldots\end{array}\right\}$ & 37.3 & 36.2 & 38.8 \\
\hline $\left.\begin{array}{l}\text { Top layer } 0^{\circ} \ldots \ldots \\
\text { Lowest layer } 90^{\circ} \ldots\end{array}\right\}$ & 31.8 & 32.2 & 31.6 \\
\hline
\end{tabular}

* LAI $=4.0,10$ layers, $38^{\circ}$ North Latitude, July 1.

\section{Problem 4-A Real Community}

Suitable data on canopy structure for simulation of community photosynthesis are provided by experiments conducted at Davis, California. Details of these experiments will be published elsewhere. Briefly, seven population densities of maize, from half a low commercial rate to three times a high rate 


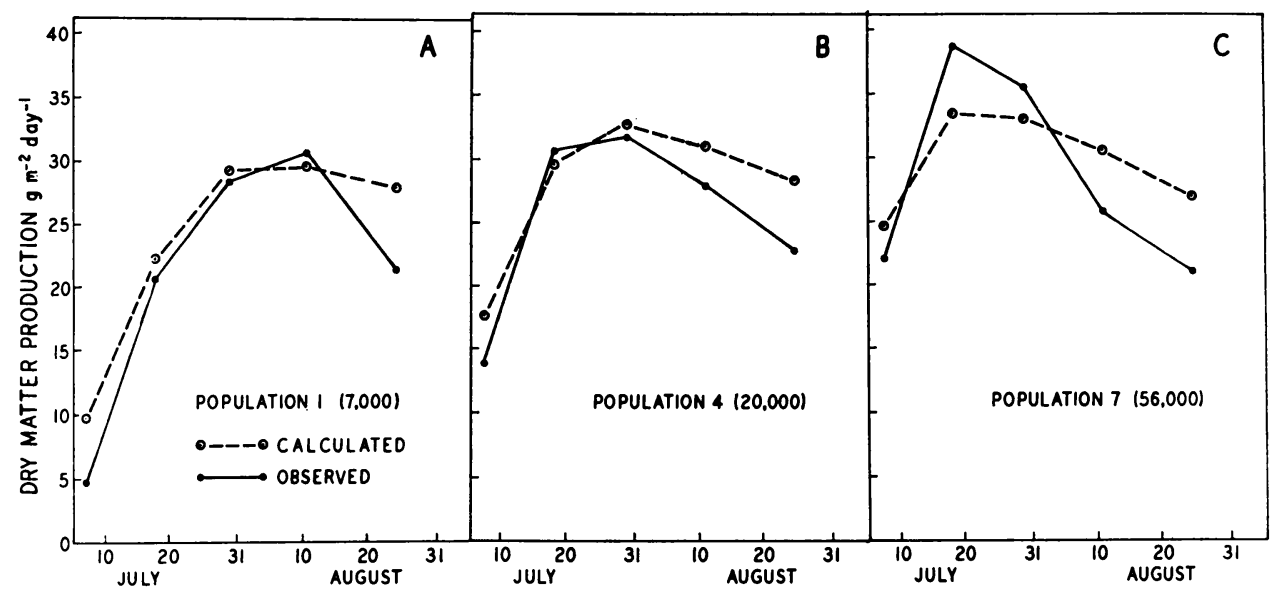

Fig. 4. Calculated and observed rates of dry matter production for maize. (A) 7,000 plants per acre; (B) 20,000 plants per acre; (C) 56,000 plants per acre.

were compared in a well-replicated field experiment. Harvests were made at approximately two-week intervals from 30 days after planting to maturity. At each harvest, representative plants were selected from each replicate of each population, and leaf area, leaf angle and spatial distributions were determined. Also, a sample of plants was harvested, dried, and weighed so that dry-matter production could be determined directly. The weather was uniformly clear during the growing period, and water and nutrients were not limiting to growth. The data on canopy structure and light environments were sufficient to permit simulation with the model for comparison with measured rates of dry-matter production. Figure 4 shows these comparisons for three of the population densities.

Correlation analysis of data for all observed and computed points in the three figures yielded a highly significant $r$ value of 0.94 (13 d.f.). Recognized errors in the assumptions would explain a part of the differences found. The assumption that respiratory losses for the whole community during a 24- hour period equal to 40 per cent of net photosynthesis is close to values used by others (Monteith, 1965; Moss et al., 1961; Loomis and Williams, 1963). However, this estimate is only approximate, and there is little reason to assume a simple, direct proportionality between photosynthesis and respiration. No experimental data were found to indicate better values. Also, light interception by tassels which should have influenced light conditions after the fourth harvest (August 5) was not measured. Shading by tassels should have reduced photosynthesis for all rates of planting, and the reduction should have been greatest for the higher populations. Another possible cause for differences was the progressive damage to leaves by wind, insects, and disease, as well as a normal decline in photasynthesis due to senescence. This should have reduced observed values below calculated values based on healthy leaf area. No allowance was made for such effects. Despite these limitations, the computer simulations are remarkably similar to the actual data both in absolute values and qualitative behavior. 


\section{APPENDIX A}

\section{The Wilson-Reeve Equations for Computing the Ratio between the Shadow Cast by a Leaf and Its Actual Area}

This material is taken directly from Reeve (1960). The wording has been modified to fit the present problem, with some of the mathematical details omitted in the interest of brevity.

To consider the problem we will assume, for reference only, that the sun is at some angle $\beta$ above the horizon, and that it may move from east to west. If we consider a system of rectangular coordinates, $O X Y Z$, in which $O X$ is due east, $O Y$ is due south and $O Z$ is vertically upward, the direction cosines of the sun's rays are proportional to $(0, \cos \beta, \sin \beta)$.

Let a small flat leaf be at the angle $\alpha$ to the horizontal, with the axis of this angle at a direction $\theta$ degrees south of east where $\theta$ can have any value between $0^{\circ}$ and $360^{\circ}$. The direction cosines of the normal to this leaf are proportional to $(\sin \alpha$ $\sin \theta,-\sin \alpha \cos \theta, \cos \alpha)$; thus its shadow area is the positive numerical value of $A(\cos \alpha \sin \beta-\sin \alpha \cos \beta \cos \theta)$.

It follows therefore, that if the directional orientation of the leaves were random, the average shadow area of a large number of such leaves would be related to the actual area by the equation:

$$
\left.F^{\prime}=F \text { [average value of }|\cos \alpha \sin \beta-\sin \alpha \cos \beta \cos \theta|\right]
$$

as $\theta$ varies from $0^{\circ}$ to $360^{\circ}$.

Reeve divided the solution into two possible conditions: the first, when the leaf angle $\alpha$ is equal to or less than $\beta$, the sun angle; the second, when $\alpha$ is greater than $\beta$. In the first case he shows that:

$$
F^{\prime} / \mathrm{F}=\cos \alpha \sin \beta \text {. }
$$

This would be the condition when sun elevations and leaf angles were such that the rays of the sun could never illuminate the lower surface of the leaf.

In the second case, when the lower leaf surface could be illuminated at some positions, the equation would be:

$$
\frac{F^{\prime}}{F}=\left[\frac{2}{\pi} \sin \alpha \cos \beta \sin \theta_{0}+\left(1-\frac{\theta_{0}}{90}\right) \cos \alpha \sin \beta\right]
$$

where the value of $\theta_{0}$ is that value of $\theta$ between $0^{\circ}$ and $90^{\circ}$ which satisfies the equation:

$$
\cos \theta=\cot \alpha \tan \beta \text {. }
$$

Using these two equations, a table of values of $F^{\prime \prime} / F^{\prime}$ was computed for every $5^{\circ}$ value of both leaf angle, $\alpha$, and sun angle, $\beta$, and given to the computer as input data for reference throughout subsequent calculations. 
TABLE A-1

RATIOS BETWEEN THE SHAIOW CAST BY A LEAF $\left(F^{\prime}\right)$ ON A PLANE NORMAL TO THE SUN'S RAYS AND THE ACTUAL AREA OF THE LEAF $(F)$

\begin{tabular}{|c|c|c|c|c|c|c|c|c|c|c|c|c|c|c|c|c|c|c|c|c|}
\hline \multirow{2}{*}{ 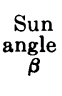 } & \multicolumn{19}{|c|}{ Leaf angle $a$} & \multirow{2}{*}{$\begin{array}{l}\text { Sun } \\
\text { angle } \\
\beta\end{array}$} \\
\hline & $0^{\circ}$ & $5^{\circ}$ & $10^{\circ}$ & $15^{\circ}$ & $20^{\circ}$ & $25^{\circ}$ & $30^{\circ}$ & $35^{\circ}$ & $40^{\circ}$ & $45^{\circ}$ & $50^{\circ}$ & $55^{\circ}$ & $60^{\circ}$ & $65^{\circ}$ & $70^{\circ}$ & $75^{\circ}$ & $80^{\circ}$ & $85^{\circ}$ & $90^{\circ}$ & \\
\hline $0^{\circ}$ & .000 & .055 & .111 & .165 & .218 & .269 & .318 & .365 & .409 & .450 & .488 & .521 & .551 & .577 & .598 & .615 & .627 & .634 & .637 & $0^{\circ}$ \\
\hline $5^{\circ}$ & .087 & .087 & .124 & .173 & .223 & .273 & .321 & .367 & .410 & .450 & .487 & .520 & .550 & .575 & .596 & .613 & .625 & .632 & .634 & $5^{\circ}$ \\
\hline $10^{\circ}$ & .174 & .173 & .171 & .199 & .240 & .284 & .328 & .371 & .412 & .450 & .486 & .517 & .546 & .570 & .590 & .606 & .618 & .625 & .627 & $10^{\circ}$ \\
\hline $15^{\circ}$ & .259 & .258 & .255 & 250 & .270 & .304 & .341 & .379 & .416 & .451 & .483 & .513 & .539 & .562 & .581 & .596 & .606 & .613 & .615 & $15^{\circ}$ \\
\hline $20^{\circ}$ & .342 & .341 & .337 & .330 & .321 & .335 & .361 & .391 & .421 & .451 & .480 & .506 & .530 & .550 & .567 & .581 & .590 & .596 & .598 & $20^{\circ}$ \\
\hline $25^{\circ}$ & .423 & .421 & .416 & .408 & .397 & .383 & .389 & .407 & .430 & .453 & .476 & .498 & .518 & .535 & .550 & .562 & .570 & .575 & .577 & $25^{\circ}$ \\
\hline $30^{\circ}$ & .500 & .498 & .492 & .483 & .470 & .453 & .433 & .432 & .442 & .457 & .473 & .489 & .504 & .518 & .530 & .539 & .546 & .550 & .551 & $30^{\circ}$ \\
\hline $35^{\circ}$ & .574 & .571 & .565 & .554 & .539 & .520 & .497 & .470 & .461 & 464 & .471 & .480 & .489 & .498 & .506 & .513 & .517 & .520 & .521 & $35^{\circ}$ \\
\hline $40^{\circ}$ & .643 & .640 & .633 & .621 & .604 & .583 & .557 & .527 & .492 & 476 & .471 & .471 & .473 & .476 & .480 & .483 & .486 & .487 & .488 & $40^{\circ}$ \\
\hline $45^{\circ}$ & .707 & 704 & 696 & 683 & 664 & .641 & .612 & .579 & .542 & .500 & .476 & .464 & .457 & .453 & .451 & .451 & .450 & .450 & 450 & $45^{\circ}$ \\
\hline $50^{\circ}$ & .766 & .763 & .754 & .740 & .720 & .694 & .663 & .628 & .587 & .542 & 492 & .461 & .442 & .430 & .421 & .416 & 412 & .410 & 409 & $50^{\circ}$ \\
\hline $55^{\circ}$ & .819 & .816 & .807 & .791 & .770 & .742 & .709 & .671 & .628 & .579 & .527 & .470 & 432 & .407 & .391 & .379 & .371 & .367 & .365 & $55^{\circ}$ \\
\hline $60^{\circ}$ & .866 & .863 & 853 & .837 & .814 & .785 & .750 & .709 & .663 & .612 & .557 & .497 & 433 & .389 & .361 & .341 & .328 & .321 & .318 & $60^{\circ}$ \\
\hline $65^{\circ}$ & .906 & .903 & .893 & .875 & .852 & 821 & .785 & .742 & .694 & .640 & .583 & .520 & 453 & .383 & .335 & .301 & .284 & .273 & .269 & $65^{\circ}$ \\
\hline $70^{\circ}$ & .940 & .936 & .925 & .908 & .883 & .852 & .814 & .770 & .720 & .664 & .604 & .539 & .470 & .397 & .321 & .270 & .240 & .223 & .218 & $70^{\circ}$ \\
\hline $75^{\circ}$ & .966 & .962 & 951 & .933 & .908 & .875 & .837 & .791 & .740 & .683 & .621 & .554 & .483 & .408 & .330 & .250 & .199 & .173 & .165 & $75^{\circ}$ \\
\hline $80^{\circ}$ & .985 & .981 & .970 & .951 & .925 & .893 & .853 & .807 & .754 & 696 & 633 & .565 & 492 & .416 & .337 & .255 & .171 & .124 & .111 & $80^{\circ}$ \\
\hline $85^{\circ}$ & .996 & .992 & .981 & .962 & .936 & .903 & .863 & .816 & .763 & .704 & .640 & .571 & 498 & .421 & .341 & .258 & .173 & .087 & .055 & $85^{\circ}$ \\
\hline $90^{\circ}$ & 1.000 & .996 & 985 & .966 & .940 & .906 & .866 & .819 & .766 & .707 & .643 & .574 & .500 & .423 & .342 & .259 & .174 & .087 & .000 & $90^{\circ}$ \\
\hline
\end{tabular}

\section{APPENDIX B}

\section{Theory for the Computation of Leaf Illumination by Skylight}

\section{Richard Hanau}

\section{The general equations}

Figure B-1 shows a general radiating source, $S$, of arbitrary shape and size. The small elemental area of this surface, $d S$, is characterized by a brightness, $B$, which is a measure of the power radiated per unit area. In general, $B$ is a function of the particular location of $d S$ on the surface, and of the direction of $r$.

The area, $A$, also of arbitrary shape and size, is a surface which receives energy from $S$. The illumination, $E$, at a small elemental area, $d A$, is a measure of the power received per unit area.

The problem here is the calculation of the illumination at the point $d A$ resulting from the entire source, $S$. The two small areas, $d S$ and $d A$, are separated by a distance, $r$. The normals to the surfaces are inclined to $r$ at the angles $\Psi$ and $\phi$. 


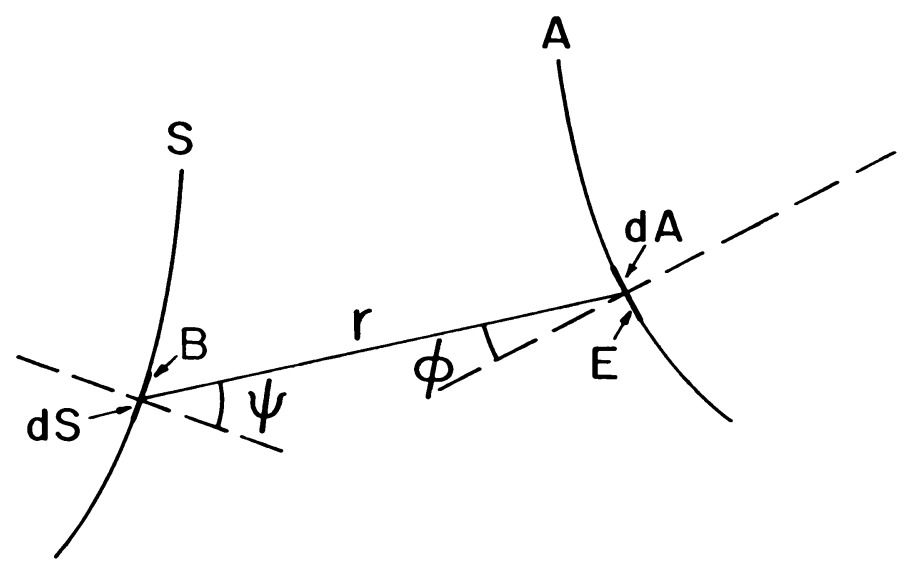

Fig. B-1. Relative orientation of the source $S$, of brightness $B$, and the illuminated area $A$.

The quantity called flux, $d F$, is a measure of the power flowing away from $d S$ and contained within a cone of solid angle, $d \Omega$. Specifically,

$$
d F=B \cos \Psi d S d \Omega .
$$

Because we are concerned with the flux incident on $d A$, we take the base of the solid angle equal to $d A$. Hence,

$$
d \Omega=\frac{\cos \phi}{r^{2}} d A
$$

The illumination at $d A$, resulting from the source $d S$, is defined as

$$
d E=\frac{d F}{d A} .
$$

Substituting equations (1) and (2) into equation (3), and indicating the integration, we have

$$
\int d E=\int \frac{B \cos \Psi \cos \phi}{r^{2}} d S=E,
$$

where $E$ is the total illumination at $d A$ due to the entire source, $S .^{1}$

\section{The specific equation}

The basic equation (4) is used to evaluate the illumination at the area $d A$ resulting from skylight. The following assumptions simplify the equation.

(a) The area $S$, here the sky, is a hemisphere of radius $r$.

(b) The brightness of the sky is constant, independent of the position of $d S$.

\footnotetext{
1 The letters and terms used here $-B$ for brightness, $E$ for illumination, and $F$ for flux-are generally more familiar than the photometric terms usually used to describe the effect of light on the human eye ( $B$ for luminance, $E$ for illuminance, and $F$ for luminous flux). The material of this appendix applies to any kind of radiant energy, and the terms can be replaced by the corresponding radiometric quantities: $N$ (radiance) for $B, H$ (irradiance) for $E$, and $P$ (radiant flux) for $F$. The final results, in any case, will be independent of the specific letters used, because ratios, often called reduced quantities, are calculated.
} 
Because of $(a), d A$ is at the center of the hemisphere, $r=$ constant, and $\Psi=0$. Because of $(b), B$ can be taken outside the integral sign. Hence,

$$
d E=\frac{B \cos \phi}{r^{2}} d S
$$

and equation (4) becomes

$$
E=\frac{B}{r^{2}} \int \cos \phi d S
$$

\section{The spherical coordinate system}

The origin of coordinates is at $d A$ (see fig. B-2). $d S$ has coordinates $(r, \delta, \rho)$, where $\delta$ is the zenith angle, varying between zero (at the zenith) and $\pi / 2$ (at the horizon); and $\rho$ is the azimuthal angle. The angle $\rho$ is measured counter-clockwise, as viewed from the zenith, and is zero at that meridan where the plane containing $d A$ is nearest to the zenith. $d A$ is inclined to the horizontal at the angle $\alpha$.

Substitution of the elemental area $d S=r^{2} \sin \delta d \delta d \rho$ into equation (5) gives

$$
E=B \iint \cos \phi \sin \delta d \delta d \rho
$$

where the double integral is to be taken over $\delta$ and over $\rho$. Cos $\phi$ must be expressed as a function of $\alpha, \delta$, and $\rho$ before integrating.

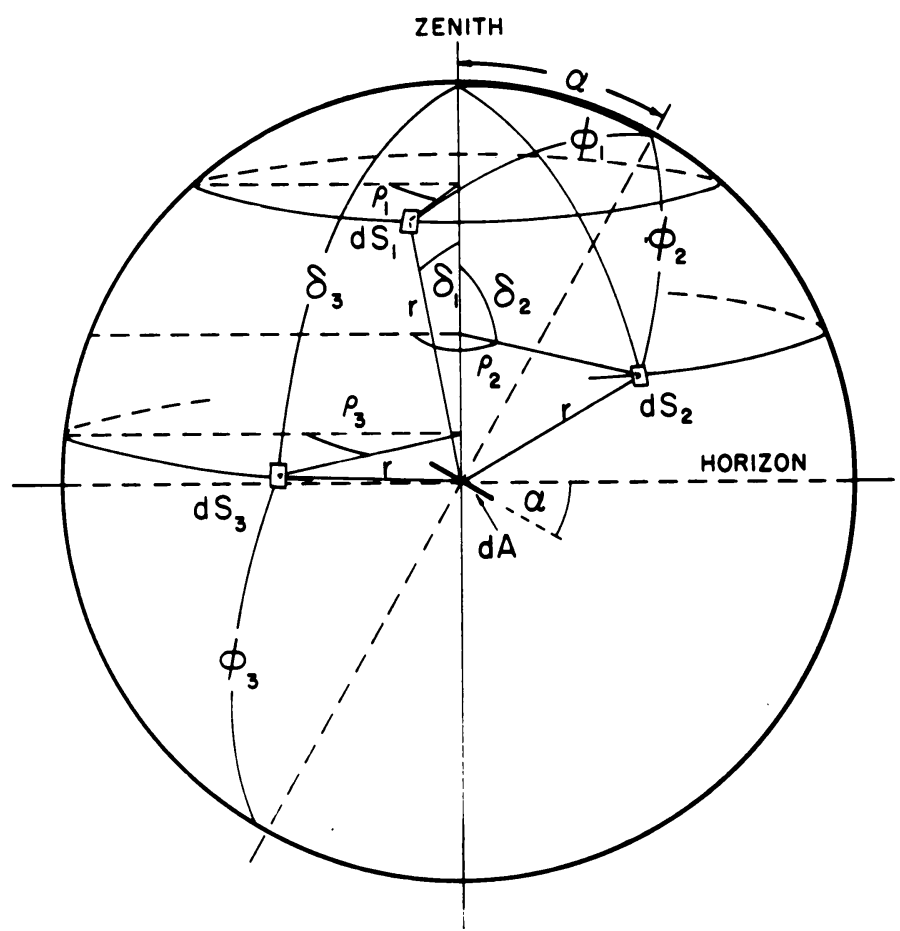

Fig. B-2. The spherical coordinate system when the source is a hemisphere and the area is at its center. 


\section{A special case: horizontal area}

The illumination for any arbitrary orientation of the area, $d A$, can be expressed conveniently relative to its value on a horizontal area. Therefore, we first use equation (6) to calculate the illumination for $\alpha=0$. In this case, since the normal to the surface is directed toward the zenith, $\delta=\phi$, and equation (6) becomes, after indicating the limits of integration,

$$
E_{o}=B \int_{o}^{\pi / 2} \int_{o}^{2 \pi} \cos \delta \sin \delta d \delta d \rho .
$$

The final result is $E_{o}=\pi B$, the subscript on $E$ indicating that $\alpha=0$.

\section{The general case: inclined area}

In the main body of this paper, Bouguer's law is stated in terms of a quantity, $I_{o}$, which is equal to the illumination $E . I_{o}$ is defined as

$$
I_{o}=\left(\frac{E}{E_{o}}\right) E_{o}=\left(\frac{E}{E_{o}}\right) \pi B=e \pi B,
$$

where $e$ is the reduced illumination. Equation (6) now becomes

$$
e=\frac{1}{\pi} \iint \cos \phi \sin \delta d \delta d \rho
$$

When the area $d A$ is inclined at an angle $\alpha \neq 0$, both the top and the bottom of the area are illuminated. The total illumination on the top of $d A$ is the sum of two parts, $e_{1}$ and $e_{2}$. The first part, $e_{1}$, is due to the sky for zenith angles $0 \leqq \delta \leqq\left(\frac{\pi}{2}-\alpha\right)$. For this part, $d S_{1}$ can vary in azimuthal angle from 0 to $2 \pi$. Using the relation between the sides of a spherical triangle, in this case the triangle $\delta, \alpha, \phi$

$$
\cos \phi_{1}=\cos \alpha \cos \delta-\sin \alpha \sin \delta \cos \rho .
$$

Substituting equation (8) into (7), and assigning integration limits on $\rho$,

$$
\pi e_{1}=2 \iint_{0}^{\pi}[\cos \alpha \cos \delta-\sin \alpha \sin \delta \cos \rho] \sin \delta d \delta d \rho
$$

The great circle at $\rho=0$ and $\rho=\pi$ is a circle of symmetry. Hence, the factor of 2 and the integration limits 0 and $\pi$ appear as given. Integrating with respect to $\rho$,

$$
\begin{aligned}
\pi e_{1} & =\left.2 \int[\rho \cos \alpha \cos \delta-\sin \alpha \sin \delta \sin \rho] \sin \delta d \delta\right|_{o} ^{\pi} \\
& =2 \pi \cos \alpha \int \sin \delta \cos \delta d \delta .
\end{aligned}
$$

In computing numerical results, it is convenient to divide the sky into circular zones between $\delta_{\min }=\delta_{i}$ and $\delta_{\max }=\delta_{a}$. These are the limits used in evaluating equation (10). The result is

$$
\pi e_{1}=\pi \cos \alpha\left(\sin ^{2} \delta_{a}-\sin ^{2} \delta_{i}\right)=\pi \cos \alpha \sin \left(\delta_{a}+\delta_{i}\right) \sin \left(\delta_{a}-\delta_{i}\right) .
$$


The second part of the illumination on the top of the area, $e_{2}$, is due to the region $\left(\frac{\pi}{2}-\alpha\right) \leqq \delta \leqq \frac{\pi}{2}$. Reference to figure B-2 will show that the integration limits on $\rho$ for $d S_{2}$ are $\rho_{\mathrm{o}}$ and $\left(2 \pi-\rho_{0}\right)$; these limits are the meridians where the plane containing $d A$ intersects the sky at the zenith angle $\delta$. Since at $\rho=\rho_{0}, \phi=\pi / 2$, equation (8) results in

$$
\cos \rho_{o}=\cot \alpha \cot \delta \text {. }
$$

Integration of equation (9), using the limits on $\rho$ applicable here, gives an equation analogous to equation (10), namely,

$$
\begin{gathered}
\pi e_{2}=\left.2 \int[\rho \cos \alpha \cos \delta-\sin \alpha \sin \delta \sin \rho] \sin \delta d \delta\right|_{\rho_{o}} ^{\pi} \\
=2 \pi \cos \alpha \int \sin \delta \cos \delta d \delta-2 \cos \alpha \int \rho_{o} \sin \delta \cos \delta d \delta+2 \sin \alpha \int \sin \rho_{o} \sin ^{2} \delta d \delta .
\end{gathered}
$$

The integration with respect to $\delta$ is evaluated by considering the three integrals in equation (12).

The first term in equation (12) is just equation (10) and has already been evaluated. The second term involves the integral

$$
I_{1}=\int \rho_{o} \sin \delta \cos \delta d \delta=\int \sin \delta \cos \delta \cos ^{-1}(\cot \alpha \cot \delta) d \delta
$$

This may be integrated first by parts. If $I_{1}=\int u d v$ and $u=\cos ^{-1}(\cot \alpha \cot \delta)$, then

$$
2 I_{1}=\sin ^{2} \delta \cos ^{-1}(\cot \alpha \cot \delta)-\cot \alpha \int \frac{d \delta}{\left(1-\cot ^{2} \alpha \cot ^{2} \delta\right)^{1 / 2}}
$$

The integral in equation (13) may be evaluated by making a change in variable, $x=\left(1+\cot ^{2} \alpha\right)^{1 / 2} \cos \delta$. The result is

$$
\begin{aligned}
I_{2} & =\int \frac{d \delta}{\left(1-\cot ^{2} \alpha \cot ^{2} \delta\right)^{1 / 2}}=-\frac{1}{\left(1+\cot ^{2} \alpha\right)^{1 / 2}} \int \frac{d x}{\left(1-x^{2}\right)^{1 / 2}} \\
& =-\sin \alpha \sin ^{-1}\left(\frac{\cos \delta}{\sin \alpha}\right) .
\end{aligned}
$$

The third term of equation (12) involves the integral

$$
\begin{aligned}
I_{3} & =\int \sin ^{2} \delta\left(1-\cot ^{2} \alpha \cot ^{2} \delta\right)^{1 / 2} d \delta \\
& =\int \sin \delta\left(\sin ^{2} \delta-\cot ^{2} \alpha \cos ^{2} \delta\right)^{1 / 2} d \delta
\end{aligned}
$$


Making the same change of variables as in $I_{2}$, equation (14) becomes

$$
\begin{aligned}
& I_{3}=-\frac{1}{\left(1+\cot ^{2} \alpha\right)^{1 / 2}} \int\left(1-x^{2}\right)^{1 / 2} d x \\
& =-\frac{\sin \alpha}{2}\left[\frac{\cos \delta}{\sin \alpha}\left(1-\frac{\cos ^{2} \delta}{\sin ^{2} \alpha}\right)^{1 / 2}+\sin ^{-1}\left(\frac{\cos \delta}{\sin \alpha}\right)\right] .
\end{aligned}
$$

We now evaluate the three terms in equation (12) at the limits $\delta_{i}$ and $\delta_{a}$. They can be simplified trigonometrically because $\rho_{o}$ is an angle in the first quadrant for any $\alpha$ and any $\delta$. The final result may be put in the form:

$$
\begin{aligned}
\pi e_{2} & =\cos \alpha\left[\sin ^{2} \delta_{a} \cos ^{-1}\left(-\cot \alpha \cot \delta_{a}\right)-\sin ^{2} \delta_{i} \cos ^{-1}\left(-\cot \alpha \cot \delta_{i}\right)\right] \\
& +\sin ^{-1}\left(\csc \alpha \cos \delta_{i}\right)-\sin ^{-1}\left(\csc \alpha \cos \delta_{a}\right) \\
& +\cos \delta_{i}\left[-\cos \left(\alpha+\delta_{i}\right) \cos \left(\alpha-\delta_{i}\right)\right]^{1 / 2} \\
& -\cos \delta_{a}\left[-\cos \left(\alpha+\delta_{a}\right) \cos \left(\alpha-\delta_{a}\right)\right]^{1 / 2} .
\end{aligned}
$$

The inverse sines and inverse cosines are evaluated as angles in the first and second quadrants, respectively.

The illumination on the underside of the area, $e_{3}$, may be evaluated in the same way as $e_{2}$. Since $\phi_{3}=\pi-\phi_{1}, \cos \phi_{3}=-\cos \phi_{1}$. The integration limits for $\rho$ are $-\rho_{0}$ and $\rho_{o}$. The equation corresponding to equation (12) is

$$
\pi e_{3}=-2 \cos \alpha \int \rho_{o} \sin \delta \cos \delta d \delta+2 \sin \alpha \int \sin \rho_{o} \sin ^{2} \delta d \delta .
$$

This involves integrals $I_{1}, I_{2}$, and $I_{3}$ which have been evaluated. The simplification proceeds as for $e_{2}$. The final result is:

$$
\begin{aligned}
\pi e_{3} & =\cos \alpha\left[\sin ^{2} \delta_{i} \cos ^{-1}\left(\cot \alpha \cot \delta_{i}\right)-\sin ^{2} \delta_{a} \cos ^{-1}\left(\cot \alpha \cot \delta_{a}\right)\right] \\
& +\sin ^{-1}\left(\csc \alpha \cos \delta_{i}\right)-\sin ^{-1}\left(\csc \alpha \cos \delta_{a}\right) \\
& +\cos \delta_{i}\left[-\cos \left(\alpha+\delta_{i}\right) \cos \left(\alpha-\delta_{i}\right)\right]^{1 / 2} \\
& -\cos \delta_{a}\left[-\cos \left(\alpha+\delta_{a}\right) \cos \left(\alpha-\delta_{a}\right)\right]^{1 / 2} .
\end{aligned}
$$

Both the inverse sines and inverse cosines are evaluated as angles in the first quadrant.

\section{Illumination resulting from the entire hemisphere}

Equations (11), (15), and (16) give expressions for the relative illumination, $e$, resulting from a circular zone between $\delta_{i}$ and $\delta_{a}$. For the total illumination, $e_{T}$, resulting from the entire hemisphere, the proper limits are used for $\delta_{i}$ and $\delta_{a}$. The results are:

$$
\begin{aligned}
& e_{1 T}=\cos ^{3} \alpha, \\
& e_{2 T}=\left(1+\cos \alpha-2 \cos ^{3} \alpha\right) / 2,
\end{aligned}
$$

and

$$
e_{3 T}=(1-\cos \alpha) / 2
$$


The total illumination on the top of the area is $e_{1 T}+e_{2 T}=(1+\cos \alpha) / 2$, which equals 1 for $\alpha=0$, in agreement with the definition of $e$. As the area $d A$ is tipped at various angles, $\alpha$, all the flux incident on $d A$ must be independent of $\alpha$, and equal to the flux incident on the area for $\alpha=0$. Since the total flux is the sum of the flux incident on the top and on the bottom, and since illumination is proportional to flux,

$$
e_{0}=e_{1 T}+e_{2 T}+e_{3 T}
$$

Using the values given in equations (17), the righthand side of equation (18) is unity, which is also the value of $e_{o}$.

\section{The case of variable brightness}

The assumption was made that $B=$ constant. Hence equations (11), (15), and (16) apply to constant brightness. However, they will also apply to the case of $B \neq$ constant, provided $B$ depends only on $\delta$. If $B$ is independent of azimuth, but depends on $\delta$, then the above equations hold for a zone for which $B=$ constant. If $B$ varies rapidly with $\delta$, then the hemisphere may have to be divided into many small zones. In all cases, however, $e$ is the ratio of the illumination resulting from a zone whose average brightness, $\bar{B}$, can be considered constant over that zone, to the illumination on a horizontal area due to the entire hemisphere of this particular average brightness, $\bar{B}$. Hence, for any known numerical value of $\bar{B}$ for a given zone, the illumination resulting from that zone (hence the illumination resulting from all zones) can be calculated.

Without too much trouble, equations can be derived for the case in which $B$ depends on $\rho$ as well as on $\delta$. Assume a small enough region, for which $B$ can be assumed constant, bounded by two meridians, $\rho_{i}$ and $\rho_{a}$, and two circles of $\delta_{i}$ and $\delta_{a}$. The integration limits are then $\rho_{i}$ and $\rho_{a}$, and the integrals over $\delta$ will be no more difficult to evaluate than those given. Only the substitution of the limit $\rho_{o}$ will lead to the involved equations discussed above; other limits, which are numbers independent of $\delta$, lead to easily integrable expressions.

\section{Summary of Appendix B}

Explicit equations are derived for the reduced illumination on the upper and lower sides of an inclined area at the center of a hemisphere. If the brightness of the hemisphere is known, the actual illumination can be calculated, provided the brightness is independent of azimuth. The procedure is outlined for dealing with the most general case in which brightness depends on azimuth as well as on zenith angle. This procedure is an obvious extension of the basic method developed.

Table B-1 gives relative values of the illumination, $e=e_{1}+e_{2}+e_{3}$, calculated by using equations (11), (15), and (16). The meaning of $e$ is given by the equation immediately preceding equation (7). $e$ is the ratio of the illumination, due to any portion of real sky whose brightness may be considered constant, on a surface at any inclination, to the illumination on a horizontal surface due to an entire hemisphere. The hemisphere is assumed to have the same brightness as that portion of the real sky considered. In table B-1, the sky portions are zones of equal area whose midlines are given as altitudes measured from the horizon. 
TABLE B-1

RATIOS OF THE ILLUMINATION ON A LEAF SURFACE INCLINED AT A GIVEN ANGLE DUE TO EQUAL-AREA SKY ZONES TO THE ILLUMINATION ON A HORIZONTAL LEAF SURFACE DUE TO THE ENTIRE HEMISPHERE OF UNIFORM BRIGHTNESS*

\begin{tabular}{|c|c|c|c|c|c|c|c|c|c|c|c|c|c|c|c|c|c|c|c|}
\hline \multirow{2}{*}{$\begin{array}{c}\text { Zone } \\
\text { midline }\end{array}$} & \multicolumn{19}{|c|}{ Leaf angle } \\
\hline & $0^{\circ}$ & $5^{\circ}$ & $10^{\circ}$ & $15^{\circ}$ & $20^{\circ}$ & $25^{\circ}$ & $30^{\circ}$ & $35^{\circ}$ & $40^{\circ}$ & $45^{\circ}$ & $50^{\circ}$ & $55^{\circ}$ & $60^{\circ}$ & $65^{\circ}$ & $70^{\circ}$ & $75^{\circ}$ & $80^{\circ}$ & $85^{\circ}$ & $90^{\circ}$ \\
\hline $73^{\circ}$ & .31 & .31 & .30 & .30 & .29 & .28 & .26 & .25 & .23 & .22 & .20 & .17 & .15 & .13 & .11 & .10 & .09 & .08 & .08 \\
\hline $49^{\circ}$ & .25 & .25 & .25 & .24 & .24 & .23 & .22 & .20 & .19 & .17 & .16 & .16 & .15 & .15 & .14 & .14 & .14 & .14 & .14 \\
\hline $36^{\circ}$ & .19 & .19 & .19 & .19 & .18 & .17 & .17 & .16 & .16 & .16 & .16 & .16 & .16 & .16 & .17 & .17 & .17 & .17 & .17 \\
\hline $25^{\circ}$ & .14 & .14 & .14 & .13 & .13 & .13 & .13 & .14 & .14 & .15 & .16 & .17 & .18 & .18 & .19 & .19 & .19 & .19 & .19 \\
\hline $15^{\circ}$ & .08 & .08 & .08 & .08 & .09 & .10 & .11 & 13 & .14 & .15 & .16 & .17 & .18 & .19 & .19 & 20 & .20 & .21 & .21 \\
\hline $5^{\circ}$ & .03 & .03 & .04 & .06 & .07 & .09 & .11 & .12 & .14 & .15 & .16 & .17 & .18 & .19 & .20 & .20 & .21 & 21 & .21 \\
\hline
\end{tabular}

* The sum of each column in the table is 1.00 , representing the total sky illumination.

\section{LITERATURE CITED}

Alberda, T., and L. Sibma

1962. Dry matter production and light interception of crop surfaces. II. Relation between growth and length of grass. Jaarb. Inst. biol. scheik. Onderz. LandbGewass. 47-58.

BLACK, J. N.

1963. The interrelationship of solar radiation and leaf area index in determining the rate of dry matter production of swards of subterranean clover (Trifolium subterraneum L.). Aust. Jour. Agr. Res. 14:20-38.

BROUGHAM, R. W.

1956. Effect of intensity of defoliation on regrowth of pasture. Aust. Jour. Agr. Res. 7: $377-87$.

Davidson, J. L., and C. M. Donald

1958. The growth of swards of subterranean clover with particular reference to leaf area. Aust. Jour. Agr. Res. 9:53-72.

Davidson, J. L., and J. R. PHILIP

1958. Light and pasture growth. In: Climatology and microclimatology. Proc. Canberra Sym. (1956). Arid Zone Research XI, UNESCO, Paris.

HeSKeth, J. D.

1963. Limitations to photosynthesis responsible for differences among species. Crop. Sci. 3:493-96.

KASANGA, H., and M. MonsI

1954. On the light transmission of leaves and its meaning for the production of matter in plant communities. Jap. Jour. Bot 14:304-24.

LeMON, E. R.

1960. Photosynthesis under field conditions. II. An aerodynamic method for determining the turbulent carbon dioxide exchange between the atmosphere and a corn field. Agron. Jour. 52:697-703.

Loomis, R. S., and W. A. Williams

1963. Maximum crop productivity: An estimate. Crop Sci. 3:67-72.

Ludwig, L. J., T. SAEKI, and L. T. Evans

1965. Photosynthesis in artificial communities of cotton plants in relation to leaf area. I. Experiments with progressive defoliation of mature plants. Aust. Jour. Biol. Sci. 18: 1103-118.

Monsi, M.

1960. Dry-matter reproduction in plants: I. Schemata of dry-matter reproduction. Bot. Mag., Tokyo 73:81-90. 
Monsi, M., and T. SAEkI

1953. ¿̈ber den Lichtfaktor in den Pflanzengesellschaften und seine Bedeutung für die Stoffproduktion. Jap. Jour. Bot. 14:22-52.

Moss, D. N., R. B. Musgrave, and E. R. Lemon

1961. Photosynthesis under field conditions. III. Some effects of light, carbon dioxide, temperature, and soil moisture on photosynthesis, respiration, and transpiration of corn.

Moss, D. N. Crop Sci. 1:83-87.

1964. Optimum lighting of leaves. Crop. Sci. 4:131-36.

MonTeith, J. L.

1962. Measurement and interpretation of carbon dioxide fluxes in the field. Neth. Jour. Agr. Sci. 10:334-46.

1965. Light distribution and photosynthesis in field crops. Ann. Bot. N.S. 29:17-37.

Nichiporovich, A. A.

1961. Properties of plant crops as an optical system. Soviet Pl. Physiol. 8:428-35.

Rabinowitch, E. I.

1945. Photosynthesis. Vol. I. Interscience Publishers, Inc. New York, 599 pp.

1951. Photosynthesis. Vol. II, Part 1. Interscience Publishers, Inc. New York. 1208 pp.

REEve, J. E.

1960. Appendix on derivation of formulae (in "Inclined Point Quadrats," by J. Warren Wilson). New Phytol. 59:1-8.

STANHill, G.

1962. The effect of environmental factors on the growth of alfalfa. Neth. Jour. Agr. Sci. $10: 247-53$.

TAKEDA, T.

1961. Studies on the photosynthesis and production of dry matter in the community of rice plants. Jap. Jour. Bot. 17:403-37.

Thomas, M. D., and G. R. HILL

1949. Photosynthesis under field conditions. In: J. Franck and W. E. Loomis, ed. Photosynthesis in plants. Iowa State University Press, Ames.

Verhagen, A. M. W., J. H. Wilson, and E. J. Britten

1963. Plant production in relation to foliage illumination. Ann. Bot. N. S. 27:627-40.

WATson, D. J.

1947. Comparative physiological studies on the growth of field crops. I. Variation in net assimilation rate and leaf area between species and varieties, and within and between years. Ann. Bot. N. S. 11:47-76.

1958. The dependence of net assimilation rate on leaf area index. Ann. Bot. N. S. 22:37-55.

Watson, D. J., and K. J. WitTs

1959. The net assimilation rates of wild and cultivated beets. Ann. Bot. N. S. 23:431-39.

Williams, W. A., R. S. Loomis, and C. R. LePLEY

1965a. Vegetative growth of corn as affected by population density. I. Productivity in relation to interception of solar radiation. Crop. Sci. 5:211-15.

1965b. Vegetative growth of corn as affected by population density. II. Components of growth, net assimilation rate and leaf area index. Crop Sci. 5:215-19.

WILSON, J. WARREN

1960. Inclined point quadrats. New Phytol. $59: 1-8$.

WIT, C. T. DE

1959. Potential photosynthesis of crop surfaces. Neth. Jour. Agr. Sci. 7:141-49.

1965. Photosynthesis of leaf canopies. Versl. landbouwk. Onderz. Ned. 663. 57 pp. 

The journal HILGARDIA is published at irregular intervals, in volumes of about 650 to 700 pages. The number of issues per volume varies.

Single copies of any issue may be obtained free, as long as the supply lasts; please request by volume and issue number from:

\author{
Agricultural Publications \\ University Hall \\ University of California \\ Berkeley, California $\mathbf{9 4 7 2 0}$
}

The limit to nonresidents of California is 10 separate titles. The limit to California residents is 20 separate titles.

The journal will be sent regularly to libraries, schools, or institutions in one of the following ways:

1. In exchange for similar published material on research.

2. As a gift to qualified repository libraries only.

3. On a subseription basis $-\$ 7.50$ a year paid in advance. All subseriptions will be started with the first number issued during a calendar year. Subscribers starting during any given year will be sent back numbers to the first of that year and will be billed for the ensuing year the following January. Make checks or money orders payable to The Regents of The University of California; send payment with order to Agricultural Publications at above address. 